\title{
ERKENNTNIS STATT ERBAUUNG: Hegels Begriff DES NATURWISSENSCHAFTLICHEN GESETZES
}

\section{Die wahre Gestalt des wissenschaftlichen Systems}

Da das Streben nach der Begründung einer wissenschaftlichen Philosophie ein entscheidendes Merkmal der Moderne ist, führt bei dem Thema »Moderne« kein Weg an Hegel vorbei. Wie man in der Phänomenologie lesen kann, war es Hegels Ziel, daran mitzuarbeiten, dass die Philosophie der Form der Wissenschaft näherkomme. Denn »die wahre Gestalt, in welcher die Wahrheit existiert, kann allein das wissenschaftliche System derselben sein «. ${ }^{1}$ Es geht um Einsicht und nicht um Erbauung. Und Hegels Werk hat maßgeblich dazu beigetragen, diesem Ziel näherzukommen.

Wenn das wahre Wissen Wissenschaft ist, dann kann es nach Hegel weder als Anschauung noch als unmittelbares Wissen des Absoluten (als Religion oder als Sein im Verständnis von »Sein desselben selbst «) existieren. Denn dann würde das Absolute nicht begriffen, sondern angeschaut und gefühlt werden, und die Forderung nach einem allgemeinen Zusammenhang würde dazu führen, »die Sonderungen des Gedankens zusammenschütten, den unterscheidenden Begriff zu unterdrücken und das Gefühl des Wesens herstellen, nicht sowohl Einsicht als Erbauung gewähren«. Zu der der Erkenntnis entgegenstehenden Erbauung heißt es: "Das Schöne, Heilige, Ewige, die Religion und die Liebe sind der Köder, der gefordert wird, um die Lust zum Anbeißen zu erwecken; nicht der Begriff, sondern die Ekstase, nicht die kalt fortschreitende Notwendigkeit der Sache, sondern die gärende Begeisterung soll die Haltung und fortleitende Ausbreitung des Reichtums der Substanz sein. $\ll^{2}$ Und etwas später im Text: »Das Leben Gottes und das göttliche Erkennen mag also wohl als ein Spielen der Liebe mit sich selbst ausgesprochen werden; diese Idee sinkt zur Erbaulichkeit und selbst zur Fadheit herab, wenn der Ernst, der Schmerz, die Geduld und Arbeit des Negativen darin fehlt. $\ll^{3}$

Für Hegel ist es die Arbeit des Begriffs, die ein wissenschaftliches System ausmacht. »Denn die Sache ist nicht in ihrem Zwecke erschöpft, sondern in ihrer Ausführung, noch ist das Resultat das wirkliche Ganze, sondern es zusammen mit seinem Werden; der Zweck für sich ist das unlebendige Allgemeine, wie die Tendenz das bloße Treiben, das seiner Wirklichkeit noch entbehrt, und das nackte Resultat ist der Leichnam, der die Tendenz hinter sich gelassen. ${ }^{4}$ Die Ausführung beinhaltet es nach Hegel, das Verhältnis des philosophischen Werkes zu anderen Denkungsarten, vor allem zu der der Naturwissenschaft, zu bestimmen. Damit wird das Wahre im wissenschaftlichen System der Philosophie nicht nur als Substanz, sondern ebensosehr als Subjekt aufgefasst und ausgedrückt. ${ }^{5}$ Es ist das System eines Fortschreitens im Sinne einer ständigen Begriffsentwicklung, die

1 G. W. F. Hegel, Phänomenologie des Geistes, in: Werke in 20 Bänden, auf der Grundlage der Werke von 1832-1845 neu edierte Ausgabe, Frankfurt/M. 1986, Bd. 3, 14 (Vorrede).

2 Ebd., 16.

3 Ebd., 24.

4 Ebd., 13.

5 Vgl. ebd., $22 \mathrm{f}$. 
den vorgängigen Begriff in dem neuen Begriff aufhebt. Es ist nicht die gärende Begeisterung, nicht die Erbauung, die den Reichtum der Substanz ausbreitet, gefordert, sondern es ist Erkenntnisarbeit vonnöten.

\section{Hegels wissenschaftliches System der Philosophie und die Naturwissenschaft}

Für Hegel hat die zur wissenschaftlichen Einsicht führende Arbeit des Begriffs bzw. die Selbstbewegung des Begriffs den Weg auch über die Naturwissenschaft und deren Gesetzesbegriff zu nehmen. Bei allem positiv Hervorzuhebenden, das Hegel auf diesem Weg erreicht hat, muss aber auch festgestellt werden, dass er die Naturwissenschaft teilweise unzutreffend charakterisierte, was seinem System abträglich ist.

Hegel übersieht nämlich - um es kurz zu sagen $-{ }^{6}$ die vom naturwissenschaftlichen Gesetz realisierte Einheit von Allgemeinem und Besonderem. Er erkennt nicht, dass dieses Gesetz den Charakter des Abstrakt-Allgemeinen überschreitet. ${ }^{7}$ Die naturwissenschaftliche Gesetzesbildung, so wie sie in der Physik (einschließlich der Mechanik) vorgenommen ist, vermag dies, weil sie auf funktionalen Abhängigkeitsverhältnissen beruht, derart dass nicht wie nach dem gewöhnlichen Bewusstsein im Wechsel des Geschehens das Allgemeine, die Gattungen, bestehen bleiben, während die Einzeldinge vergehen, sondern das Allgemeine nur durch das Einzelne - das seinerseits nicht etwas Zufälliges, sondern durch das Ganze in seinem Charakter bestimmt ist - lebt, das Besondere hier kein für sich Bestimmtes mehr ist, das nur als Beispiel neben dem Allgemeinen besteht. Diese Art naturwissenschaftlicher Gesetzlichkeit erreicht allerdings nicht die Stufe des Konkret-Allgemeinen der Philosophie. Denn ihre Einzelne sind in der Gestalt von Messgrößen auftretende Artefakte und die jeweils fixierte Abhängigkeitsbeziehung, ihr Allgemeines, ist nicht universal, sondern gilt im Rahmen einer bestimmten Theorie, die die Rolle des Ganzen übernimmt und das naturwissenschaftlich-physikalische System ausmacht. ${ }^{8}$

Die naturwissenschaftliche Form des Allgemeinen nimmt Hegel deshalb nicht hinreichend wahr, weil er nicht die Denkweise physikalischer Theorien wie die der Newtonschen Mechanik, sondern deren philosophische Interpretation in der mechanistischen Weltansicht bestimmt (und zu Recht kritisiert). Damit hat er eine eingeschränkte Sicht auf den Charakter von Verhältnisbestimmungen, auf die es im naturwissenschaftlichen Denken tatsächlich ankommt. Das zeigt sich vor allem in seiner Diskussion des Infinitesimalkalküls und seinem Vergleich von mechanischen Gesetzen und der wissenschaftlichen Fassung des Organischen.

6 Ausführlicher dazu: H.-H. v. Borzeszkowski und R. Wahsner, Die Fassung der Welt unter der Form des Objekts und der philosophische Begriff der Objektivität, Preprint 391 des Max-Planck-Instituts für Wissenschaftsgeschichte, Berlin 2010; R. Wahsner, Der Widerstreit von Mechanismus und Organismus. Kant und Hegel im Widerstreit um das neuzeitliche Denkprinzip und den Status der Naturwissenschaft, Hürtgenwald 2006, insbes. 217-230.

7 Vgl. z. B. G. W. F. Hegel, Phänomenologie des Geistes, a. a. O., 107-136.

8 Ausführliche dazu: R. Wahsner, »Das naturwissenschaftliche Gesetz. Hegels Rezeption der neuzeitlichen Naturbetrachtung in der Phänomenologie des Geistes und sein Konzept von Philosophie als Wissenschaft«, in: Hegel-Jahrbuch 2001. Phänomenologie des Geistes. Erster Teil, hg. v. A. Arndt, K. Bal, H. Ottmann, in Verbindung mit D. Rodin, Berlin 2002, 172-178; H.-H. v. Borzeszkowski, "Zum Status des induktiven Vorgehens in Hegels Begriff der beobachtenden Vernunft«, in: ebd., 179; H.-H. v. Borzeszkowski und R. Wahsner, Die Fassung der Welt unter der Form des Objekts und der philosophische Begriff der Objektivität, a. a. O. 
Eine Analyse des Hegelschen Verständnisses der naturwissenschaftlichen Begrifflichkeit hat ergeben, ${ }^{9}$ dass Hegel bei aller Wertschätzung des Infinitesimalkalküls den Zusammenhang zwischen diesem Kalkül und dem Begriff der Bewegung, mithin auch nicht dessen Bedeutung für die physikalische Bewegungsfassung erkennt. Dadurch bleiben ihm Einsichten in wesentliche Aspekte dieses Kalküls und des Status der Naturwissenschaft verschlossen. Er arbeitet zwar heraus, dass das mathematische Unendliche des Infinitesimalkalküls dem wahrhaft Unendlichen der Philosophie nahesteht. Da er aber die physikalische bzw. naturwissenschaftliche Denkweise mit der mechanistischen identifiziert, bleibt ihm verschlossen, dass die Physik (schon in der Mechanik) vermittels dieses Kalküls die Bewegung zu erfassen vermag.

Im Abschnitt »Beobachtende Vernunft« der Phänomenologie, in dem Hegel den Unterschied von Organischem und Unorganischem betrachtet, wird seine unzureichende Auffassung ebenfalls sehr deutlich. Nach Ausführungen über die Begrifflichkeit des (mechanischen) Gesetzes, die den Status des physikalischen Gesetzes teilweise treffend charakterisieren, vermeint Hegel in der Bestimmtheit der Begriffe, etwa der der Physik, eine Grenze des Unorganischen zu erkennen. Das zeigt sich vor allem in den Passagen, in denen er erklärt, warum die beobachtende Vernunft im Organischen des Zweckbegriffs bedarf: »Allein wie vorhin das Organische bestimmt worden, ist es in der Tat der reale Zweck selbst; denn indem es sich in der Beziehung auf Anderes selbst erhält, ist es eben dasjenige natürliche Wesen, in welchem die Natur sich in den Begriff reflektiert, und die an der Notwendigkeit auseinandergelegten Momente einer Ursache und einer Wirkung, eines Tätigen und eines Leidenden in eins zusammengenommen, so daß hier etwas nicht nur als Resultat der Notwendigkeit auftritt; sondern, weil es in sich zurückgegangen ist, ist das Letzte oder das Resultat, ebensowohl das Erste, welches die Bewegung anfängt, und sich der Zweck, den es verwirklicht. ${ }^{10}$ Ohne behaupten zu wollen, dass der Gesetzesbegriff der unorganischen Physik ausreicht, um das Organische gesetzmäßig zu fassen, muss darauf verwiesen werden, dass die Grenze zwischen Organischem und Unorganischem wegen einer Verkennung des physikalischen Gesetzesbegriffs von Hegel nicht adäquat bestimmt wird. ${ }^{11}$ Das ist insofern besonders misslich, als es Hegels erklärtes Ziel war, herauszufinden, "wie das Denken zunächst in der Physik ist «. ${ }^{12}$

Hegel hat zwar, indem er die Mechanik - so wie er sie verstand - kritisch beleuchtete und auf den Begriff bringen wollte - indirekt viel Zutreffendes über die in der Mechanik (und damit über die in der durch sie geprägten Physik) schon de facto vorhandene Denkungsart gesagt, da er aber das Zutreffende der Physik nicht selbst zugestand, sein Ziel nicht erreicht, das Verhältnis des philosophischen Werkes zu anderen Wissenschaften zu bestimmen.

\section{Die postmoderne Gefährdung der Wissenschaft}

Die Wissenschaftlichkeit des philosophischen Systems gründet maßgeblich auf der (anders gearteten, aber ebenfalls systematisch verfassten) Wissenschaftlichkeit der Fachwissenschaft, insbesondere auf der der Naturforschung. Damit ist allerdings auch klar, dass es verschiedenartige Gefährdungen

9 Vgl. H.-H. v. Borzeszkowski und R. Wahsner, »Infinitesimalkalkül und neuzeitlicher Bewegungsbegriff oder Prozeß als Größe«, in: Jahrbuch für Hegelforschung 2002, hg. v. H. Schneider, Sankt Augustin 2004, 197-271.

10 Vgl. G. W. F. Hegel, Phänomenologie des Geistes, a. a. O., 198.

11 Ausfuhrlicher dazu: H.-H. v. Borzeszkowski, »Kann die Physik das Leben wissenschaftlich erfassen? « in: Hegel-Jahrbuch 2006. Das Leben denken. Erster Teil, hg. v. A. Arndt, P. Cruysberghs, A. Przylebski, unter Mitwirkung von F. Fischbach, Berlin 2006, 197-201.

12 G. W. F. Hegel, Enzyklopädie. Zweiter Teil. Die Naturphilosophie, in: Werke, a. a. O., Bd. 9, 11 (Einleitung). 
der Philosophie gibt. Die erste entsteht, wenn die Philosophie meint, sich nicht auf das Faktum Wissenschaft stützen zu müssen. Dann herrscht - um es mit Cohen zu sagen - das »Faustrecht der Spekulation $\ll .{ }^{13}{ }$ Es bleibt alsdann ein Phantasieren über allgemeine Fragen des menschlichen Daseins übrig, in dessen stilistischer Darstellung ein poetischer Schwung und eine gewisse plastische Kraft über die Mängel und Blößen, ja sogar über das gänzliche Fehlen begrifflicher Erörterungen hinwegtäuschen kann. « ${ }^{14}$ Die Wissenschaftlichkeit der Philosophie ist aber auch gefährdet, wenn die Philosophie sich zwar auf die Wissenschaft stützen will, deren Erkenntnisart aber missversteht - wie es nach oben Gesagtem zum Teil bei Hegel zu finden ist - oder wenn die Naturforschung gar selbst ihren wissenschaftlichen Weg verlässt und postmoderne Züge annimmt.

Die Gefahr, dass die Naturforschung sich selbst ihres wissenschaftlichen Charakters beraubt oder diesen zumindest verwässert, ist durchaus gegeben, so dass die Natur»wissenschaft« zusammen mit der sich auf sie stützenden Philosophie zur faden Erbaulichkeit herabzusinken droht.

Das wird schon bei einem Blick auf das Angebot an populärer Literatur über Themen aus der Quantentheorie und der Allgemeinen Relativitätstheorie deutlich. Er zeigt nämlich, dass es sich dabei nicht nur um die üblichen, von Journalisten und anderen physikalischen Laien verfassten, oft missverständlichen Texte handelt, sondern auch um die von anerkannten Spezialisten ihres Fachs vorgelegten Darstellungen. Da werden Begriffe aus der physikalischen Disziplin Quantentheorie verwendet, um eine esoterische Subjekt-Objekt-Mystik zu entwickeln und phantastische Geschichten über das Erleben der Subjekte in einer von mikroskopischen Gesetzen beherrschten Welt zu erzählen, oder man würdigt Begriffe aus der Relativitätstheorie zu Termini herab, die es ermöglichen, eine dem unmittelbaren Erleben des Lesers scheinbar zugängliche phantastische Welt der raum-zeitlichen Wurmlöcher und der Schwarzen Löcher als dunkle Fallen der Raum-Zeit aufzubauen - ganz zu schweigen von den Elaboraten über den Kosmos. Das wird - wie gesagt - oft von renommierten Fachleuten verfasst, und man muss annehmen, dass dies nicht nur dem Kommerz zuzuschreiben ist. Zwar wird auch der eine Rolle spielen, denn Infotainment, also Wissenschaftsunterhaltung verkauft sich besser als ein auf wissenschaftliche Information gerichteter und daher manchmal auch anstrengender Text. Aber es ist wohl schlimmer: Diese aus den Fachdisziplinen kommenden Autoren meinen es ernst, was sie darin über Grundlagen, Themen und Perspektiven ihres Fachs darlegen, und dokumentieren damit den Verfall des Verständnisses für den Geist der Physik. Sie zeigen den Einbruch postmodernen Denkens in die Physik, was von ihnen manchmal sogar ausdrücklich begrüßt wird. Zum Beispiel kann man auf dem Klappentext eines derartigen Buches den Satz finden, dass dieses Werk »eine existentielle Bereicherung für unser postmodernes Zeitalter darstellt«. ${ }^{15}$

Die sich in der Popularisierung zeigende Auffassung der Physik berücksichtigt nicht, dass es in der Naturwissenschaft ebensowenig wie in der Philosophie um Erbauung gehen darf. Und diese Auffassung hat es früher auch nicht in diesem Ausmaß gegeben, was durch einen Vergleich mit populären Darstellungen älteren Datums belegt werden kann. Man denke nur an die populären Vorträge und Schriften eines Boltzmann, Planck, Einstein, Schrödinger, Heisenberg usw., in denen versucht wurde, die Prinzipien dieser Wissenschaft verständlich zu machen. ${ }^{16}$ Zwar wurden auch da manchmal

$13 \mathrm{H}$. Cohen, »Einleitung mit kritischem Nachtrag zur Geschichte des Materialismus von F. A. Lange«, in: Hermann Cohen, Werke, hg. vom Hermann-Cohen-Archiv unter der Leitung von Helmut Holzhey, Hildesheim, Zürich, New York 1984, Bd. 5, 10.

14 Ebd., 19.

15 Es handelt sich um das Buch von John D. Barrow Die Entdeckung des Unmöglichen (Heidelberg, Berlin, Oxford 1998), wobei der zitierte Text einer Besprechung desselben in dem Journal Physik in unserer Zeit entnommen ist.

16 Selbst dann, wenn kommerzielle Überlegungen eine Rolle spielten, verriet man die Physik nicht an den gewöhnlichen Geschmack. So kam das Buch Die Evolution der Physik von A. Einstein und L. Infeld 
die Grenzen der Physik überschritten. Diese Darstellungen, die die Physik (z. B. in dem Versuch, allein mit der Physik zu einem geschlossenen Weltbild zu kommen) überdehnten, waren aber keineswegs physikalische Prinzipien und Ergebnisse mystifizierende, der Erbauung dienende Texte, sondern zumeist und zuvorderst darauf gerichtet, die Reichweite der Physik und deren Geist herauszuarbeiten und gebildeten Laien zugänglich zu machen.

Die Zersetzungstendenzen in der Physik, die sich symptomatisch in der oben angesprochenen Literatur zeigen, werden dadurch befördert, dass die technischen Möglichkeiten es gestatten, gewisse Aspekte physikalischer Lösungen von Grundgesetzen und von Modellen, die auf dieser Grundlage konstruiert werden, mittels Computerprogrammen zu visualisieren. Diese Computermodelle scheinen die Physik anschaulich zu machen, und indem die Begeisterung unter den Wissenschaftlern dafür wächst, gewinnen zunehmend die wissenschaftlichen Programme die Oberhand, welche die jeweils neueste Hard- und Software auszunutzen gestatten. Computer- und andere Firmen nutzen das aus und fördern gerade die Projekte, für welche die jeweils neueste Computertechnik (bzw. allgemeiner, neuestes High-tech-Gerät) gekauft werden muss. Dabei bleibt die eigentliche physikalische Grundlagenforschung zunehmend auf der Strecke, da die Technik nicht mehr als sehr nützliches Hilfsmittel, sondern indirekt als wissenschaftliche Programme bestimmender Akteur auftritt.

Die Wissenschaftler, in Sonderheit auch die Physiker, sind nicht nur Verführte, sondern auch Aktivisten in dieser Entwicklung. Einerseits werden sie dazu gedrängt, so zu arbeiten, andererseits treiben sie diese Entwicklung mit ihrer Arbeit selbst voran, indem sie an die wissenschaftliche Bedeutung der Bilder (als Dokumente der Objektivität) glauben, an Bilder, die oft sowohl in einer Fachzeitschrift als auch im Feuilleton einer Tageszeitung oder einem populärwissenschaftlichen Buch Verwendung finden können. Die schwere Arbeit der Gesetzeserkenntnis, das Verstehen von Prinzipien und deren Reichweite überfliegt man so und glaubt schließlich, aus eigenem Ansehen die physikalischen Prozesse zu verstehen. Das führt von dem weg, was die Physik ausmacht, und nimmt ihr die Erkenntnisfunktion. Es macht sie zu einem Sammelsurium von Bildern und Erzählungen, deren Zusammenhang nicht durch Naturprinzipien in Form eines Systems von grundlegenden Bewegungsgesetzen gegeben ist, sondern durch oberflächliche Ähnlichkeiten, die mehr durch die Art der jeweils herangezogenen Verarbeitungstechnik bestimmt werden als durch deren naturgesetzliche Gemeinsamkeiten. Selbst wenn eine große Theorie hinter den Bildern steht - wie z. B. im Fall der Kosmologie, deren Grundlage die Allgemeine Relativitätstheorie ist, - gewinnen bunte Bilder zunehmend ein Eigenleben, was dazu führt, dass man bunte »Komplettbilder des Kosmos« veröffentlicht.

Um Hegel zu paraphrasieren, es werden die Sonderungen des Gedankens zusammengeschüttet, der unterscheidende Begriff wird unterdrückt und das Gefühl des Wesens erzeugt; nicht Einsicht, sondern Erbauung wird gewährt. Wenn das zur Regel der Naturforschung wird, dann ist es um deren Wissenschaftlichkeit geschehen.

\section{Resümee}

Da es gemäß der Hegelschen Einsicht darum geht, das Verhältnis der Philosophie zur Wissenschaft, insbesondere zur Naturwissenschaft zu bestimmen und die Grenze derselben zu verstehen, um so über diese Grenze hinauszugehen und zum wissenschaftlichen System der Philosophie zu kommen, ist dieses philosophische System auch durch die Naturwissenschaft und deren Grenzen bestimmt. Daher ist das wahre Ganze des wissenschaftlichen Systems, zu dem die Philosophie in ihrer Ent-

zwar dadurch zustande, dass Einstein seinem mittellosen jungen Mitarbeiter Infeld über die Tantiemen Geld zukommen lassen wollte. Dadurch entstand dann aber eben ein ernsthaftes, die Physik nicht verballhornendes, sondern sie verständlicher machendes Werk. 
wicklung, im Prozess des Sichselbstwerdens kommen muss, von dem abhängig, was Naturwissenschaft ist und worin ihre Grenze besteht. Es gereicht also zum Schaden des philosophischen Systems, wenn diese Grenze nicht angemessen bestimmt wird oder wenn die Naturforschung weiter durch postmoderne Einflüsse ihren wissenschaftlichen Charakter dem Bedürfnis nach Erbauung opfert. Die Chance für eine wissenschaftliche Philosophie wäre so schon in ihrem Ansatz vertan.

Prof. Dr. Horst-Heino v. Borzeszkowski

Technische Universität Berlin, Institut für Theoretische Physik

Hardenbergstrasse 36, D-10623 Berlin, Germany

borzeszk@itp.physik.tu-berlin.de 